\title{
The CONTIN algorithm and its application to determine the size distribution of microgel suspensions
}

\author{
A. Scotti, ${ }^{1,2}$ W. Liu,,${ }^{2,3}$ J. S. Hyatt, ${ }^{2}$ E. S. Herman, ${ }^{4}$ H. S. Choi, ${ }^{5}$ J. W. Kim, ${ }^{6,7}$ L. A. Lyon, ${ }^{4}$ \\ U. Gasser, ${ }^{1}$ and A. Fernandez-Nieves ${ }^{2}$ \\ ${ }^{1}$ Laboratory for Neutron Scattering and Imaging, Paul Scherrer Institute, 5232 Villigen, Switzerland \\ ${ }^{2}$ School of Physics, Georgia Institute of Technology, Atlanta, Georgia 30332, USA \\ ${ }^{3}$ School of Electrical and Electronic Engineering, Shandong University of Technology, 255049 Zibo, China \\ ${ }^{4}$ School of Chemistry and Biochemistry, Georgia Institute of Technology, Atlanta, Georgia 30332, USA \\ ${ }^{5}$ Shinsegae International, 422 Apgujeong-ro, Gangnam-gu, Seoul 135-954, South Korea \\ ${ }^{6}$ Department of Applied Chemistry, Hanyang University, Ansan, Gyeonggi-do 426-791, South Korea \\ ${ }^{7}$ Department of Bionano Technology, Hanyang University, Ansan, Gyeonggi-do 426-791, South Korea
}

(Received 16 January 2015; accepted 14 May 2015; published online 17 June 2015)

\begin{abstract}
We review a powerful regularization method, known as CONTIN, for obtaining the size distribution of colloidal suspensions from dynamic light scattering data. We show that together with the so-called L-curve criterion for selecting the optimal regularization parameter, the method correctly describes the average size and size distribution of microgel suspensions independently characterized using small-angle neutron scattering. In contrast, we find that when using the default regularization process, where the regularizer is selected via the "probability to reject" method, the results are not as satisfactory. (C) 2015 AIP Publishing LLC. [http://dx.doi.org/10.1063/1.4921686]
\end{abstract}

\section{INTRODUCTION}

Microgels are cross-linked polymer networks of colloidal size immersed in a solvent. ${ }^{1}$ They respond reversibly to changes in their environment by changing their size. ${ }^{2}$ Hence, they are responsive and potentially useful in, for example, drug delivery applications. ${ }^{3}$ Similarly to other types of colloidal suspensions, the phase behavior of a microgel suspension depends on the fraction of space occupied by the particles, or volume fraction, which is determined by the particle size and particle number density. Additionally, the suspension polydispersity is known to also play a major role. In fact, sufficiently polydisperse suspensions are unable to crystallize. ${ }^{4-7}$ Therefore, reliable methods for determining both the average particle size and the size distribution of colloidal suspensions are of paramount importance.

One of the most common techniques for measuring the size of colloidal particles is light scattering, whereby a laser impinges on a dilute suspension and the scattered intensity is monitored as a function of time and scattering angle. Other techniques include sedimentation methods,${ }^{8}$ electro-resistance counting methods, ${ }^{9}$ ultrasonic attenuation spectroscopy, ${ }^{10}$ and image analysis. ${ }^{11}$

Sedimentation is a classic particle sizing technique based on the Navier-Stokes settling equation. It is often divided into two categories: gravitational and centrifugal. In either case, the particles in the suspension settle or cream under the action of gravity or centrifugal forces. From measurements of the particle settling velocity, the average particle size can be determined. The method of electro-resistance counting relies on measuring the momentary changes in electrical conductivity as the particles in a suspension pass through an orifice of appropriate size. Since the conductivity decreases whenever a non-conducting particle passes through, the size of the conductivity spike can be used to determine the particle dimensions. In ultrasonic attenuation spectroscopy, an ultrasound wave passing through the suspension is attenuated in ways that depend on the particle size. Hence, by using an appropriate model for this attenuation to fit the experimental data, the particle size can be deduced. Finally, optical imaging, as well as scanning or transmission electron imaging, all provide reasonably accurate values of the particle size and shape, if used on particles in the appropriate size range.

With light scattering, the size of the particles can be determined by either measuring the average scattered intensity as a function of the scattering angle, in what is referred to as static light scattering, or by monitoring the timefluctuations of the scattered intensity at a given scattering angle, in what is referred to as dynamic light scattering (DLS). ${ }^{12}$ The latter is most often employed for additionally determining the suspension size distribution or some measure of its polydispersity. When the sample is illuminated by a laser beam, the scattered field takes the form of a speckle pattern due to interference of light scattered by all particles in the scattering volume. The interference of the individual fields can be constructive or destructive, producing areas of high and low intensity, respectively. However, due to singleparticle diffusion, the speckle pattern is not stationary but fluctuates randomly in time. In DLS, one focuses on a single speckle and determines the time correlation function of the scattered intensity. This is done at a certain scattering angle and incident light wavelength, and hence for a certain value of the modulus of the scattering wave vector, $q$. The decorrelation of the measured intensity fluctuations results from the diffusion of the particles over length scales $\sim 1 / q$. Hence, the method naturally allows the determination of the particle diffusion coefficient, and therefore of the particle radius, via the StokesEinstein equation. ${ }^{13}$ 
The scattered intensity, $I$, is detected with a fast photon detector, such as a photomultiplier tube or an avalanche photodiode (APD), and fed into a correlator that determines the intensity correlation function, $\langle I(t) I(t+\tau)\rangle$, with $\tau$ the correlation or delay time. It is the analysis of this correlation function that allows the average particle size and suspension size distribution to be obtained.

The method of cumulants ${ }^{14}$ is widely used for this purpose. It is based on the Taylor expansion of the logarithm of the correlation function and provides an average particle size and an estimate of the width of the size distribution. However, the latter can only be obtained for suspensions that are quite monodisperse to begin with. For more general situations, the only way to obtain the particle size distribution is by performing an inverse Laplace transform of the measured correlation function. However, this problem is mathematically ill-posed: there will in general be infinitely many possible solutions, and any small change in the experimental data can cause a large change in the solution. To minimize the influence of these issues, the particle size distribution must be constrained in some way. We may, for example, require that the size distribution be larger than zero and that it goes to zero at the boundaries of the relevant size range. Physical considerations also supply constraints, although they are generally insufficient to overcome the ill-posed nature of the problem.

There are numerous approaches to solve the inversion problem involved in the determination of the particle size distribution from DLS data. Some of them include the exponential sampling method, ${ }^{15}$ the non-negative least squares algorithm, ${ }^{16}$ the truncated single value decomposition, ${ }^{17}$ the Tikhonov regularization method, ${ }^{18}$ and the neural network approach. ${ }^{19}$ The most popular and most widely used method is CONTIN, a type of Tikhonov regularization originally encoded in FORTRAN by Provencher ${ }^{20}$ and more recently emulated in MATLAB by Marino. ${ }^{21}$ Interestingly, this method also finds applicability in other contexts, including nuclear magnetic resonance. ${ }^{22}$ CONTIN uses the probability to reject regularization method. However, as Provencher recognized in his original paper, ${ }^{20}$ often the inversion problem cannot be solved with this method without requiring additional constraints on the solution; these usually rely on prior knowledge about the suspension size distribution itself. This renders the method less robust, since obtaining the correct solution to the inversion problem requires foreknowledge of the solution itself, and thus cannot be applied to the characterization of an unknown system. Other regularization strategies include the generalized cross-validation scheme, ${ }^{23}$ the quasi-optimality criterion, ${ }^{24}$ and the so-called V-curve ${ }^{25}$ and L-curve ${ }^{26}$ criteria. However, not all of these criteria have been tested rigorously in experiments.

In this paper, we show that the CONTIN method, together with the L-curve criterion, provides a reliable way to obtain the size distribution of colloidal suspensions. We start by reviewing the method in detail, emphasizing its key aspects. We then discuss the suspensions we use for testing the accuracy of the inversion method; we employ relatively monodisperse microgel suspensions and mixtures of these. We then present an independent determination of the size distribution of these suspensions using small-angle neutron scattering (SANS) and compare the results with those obtained by DLS. These agree well with each other and with what we obtain using the method of cumulants. Further, we compare the L-curve and the probability to reject methods for choosing the optimal regularizer, testing for robustness under changes in the range of decay rates considered in the fit, the point density, the experimental measurement time, which correlates with the amount of noise in the DLS data, and the scattering angle. We find that CONTIN together with the L-cruve criterion provides a robust strategy to obtain the size distribution from inverted DLS data.

\section{THEORY}

In a DLS experiment, one measures the normalized intensity time correlation function, $g^{(2)}(q, \tau)$, at a certain $q$ as a function of the correlation time, $\tau$. For ergodic systems, $g^{(2)}(q, \tau)$ can be written in terms of the field correlation function, $g^{(1)}(q, \tau)$, according to the Siegert relation ${ }^{27}$

$$
g^{(2)}(q, \tau)=\frac{\langle I(q, t) I(q, t+\tau)\rangle}{\langle I(q, t)\rangle^{2}}=1+\beta\left|g^{(1)}(q, \tau)\right|^{2},
$$

where $\langle I(q, t)\rangle$ is the mean scattered intensity and $\beta(q)$ $=g^{(2)}(q, \tau=0)-1$ defines the intercept, a measure of the experimental signal-to-noise ratio, with contributions from device alignment imperfections ${ }^{28}$ and the presence of multiple scattering. For diffusive, strictly monodisperse suspensions, the field correlation function decays exponentially with $\tau: g^{(1)}(q, \tau)=\exp (-\Gamma \tau)$, where $\Gamma=D q^{2}$ is the decay rate, with $D$ the translational single-particle diffusion coefficient and $q=\left(4 \pi n / \lambda_{0}\right) \sin (\theta / 2) ; \lambda_{0}$ is the laser wavelength in vacuum, $n$ is the refractive index of the solvent, and $\theta$ is the scattering angle. From $D$, we can obtain the hydrodynamic particle radius, $R_{h}$, using the Stokes-Einstein relation ${ }^{27}$

$$
R_{h}=\frac{k_{B} T}{6 \pi \eta D}=\frac{k_{B} T q^{2}}{6 \pi \eta \Gamma},
$$

where $k_{B}$ is Boltzmann's constant, $T$ is the absolute temperature, and $\eta$ is the dynamic viscosity of the solvent.

For the general case of polydisperse suspensions, $g^{(1)}(q, \tau)$ can no longer be represented as a single exponential and must be expressed by an integral over the decay rate. For the case of Rayleigh scattering, where $q R_{h} \lesssim 1 / 2, g^{(1)}(q, \tau)$ can be written as ${ }^{14}$

$$
g^{(1)}(q, \tau)=\int_{0}^{\infty} d \Gamma G(\Gamma) \exp (-\Gamma \tau)
$$

with $G(\Gamma)$ the decay rate distribution, which is normalized to one so that $\int_{0}^{\infty} G(\Gamma) d \Gamma=1$.

The simplest method for analyzing DLS data is the method of cumulants, whereby $\ln \left[g^{(1)}(q, \tau)\right]$ is written as a Taylor series in the correlation time, ${ }^{14}$

$$
\begin{aligned}
\ln \left[g^{(1)}(q, \tau)\right] & =\sum_{m=1}^{\infty} \frac{k_{m}}{m !}(-\tau)^{m} \\
& =-k_{1} \tau+\frac{k_{2}}{2} \tau^{2}+\cdots
\end{aligned}
$$


The coefficients in the expansion, which is in most cases truncated to second order, are called cumulant coefficients; they generally depend on the details of the decay rate distribution. For short times, the behavior is that of monodisperse suspensions, with $\ln \left[g^{(1)}(q, \tau)\right]$ linear in $\tau$, but with a slope that is equal to the mean decay rate, ${ }^{29} \bar{\Gamma}=\int_{0}^{\infty} G(\Gamma) \Gamma d \Gamma$. Hence, the expansion in Eq. (4) is truncated to linear order, with $k_{1}=\bar{\Gamma}$; from $\bar{\Gamma}$, the mean particle radius follows from Eq. (2). For longer correlation times, the above linearity is lost. We can account for this deviation when $G(\Gamma)$ is sharply peaked. In this case, we write

$$
\begin{aligned}
\exp (-\Gamma \tau) & =\exp (-\bar{\Gamma} \tau) \exp [-(\Gamma-\bar{\Gamma}) \tau] \\
& =\exp (-\bar{\Gamma} \tau)\left[1-(\Gamma-\bar{\Gamma}) \tau+\frac{(\Gamma-\bar{\Gamma})^{2}}{2 !} \tau^{2}+\cdots\right]
\end{aligned}
$$

where we have Taylor expanded the second exponential in the right hand side of this equation around the mean value $\bar{\Gamma}$. By further neglecting terms of order 3 and above, Eq. (3) becomes

$$
\begin{aligned}
g^{(1)}(q, \tau) \approx & \exp (-\bar{\Gamma} \tau) \\
& \times \int_{0}^{\infty} G(\Gamma)\left[1-(\Gamma-\bar{\Gamma}) \tau+\frac{(\Gamma-\bar{\Gamma})^{2}}{2 !} \tau^{2}\right] d \Gamma \\
= & \exp (-\bar{\Gamma} \tau)\left[1+\frac{k_{2}}{2 !} \tau^{2}\right]
\end{aligned}
$$

where $k_{2}=\int_{0}^{\infty} G(\Gamma)(\Gamma-\bar{\Gamma})^{2} d \Gamma$ is the variance of the distribution. Taking the natural logarithm, expanding $\ln [1$ $\left.+k_{2} \tau^{2} / 2\right]$ around $\tau=0$ and keeping terms up to second order, which again assumes that $G(\Gamma)$ is sharply peaked, we obtain Eq. (4). The relative polydispersity of the suspension then follows from the first and second cumulants as $\sqrt{k_{2} / k_{1}^{2}}$. We emphasize that this result is valid when $G(\Gamma)$ is sharply peaked: when $\sqrt{k_{2} / k_{1}^{2}} \lesssim 0.08$. $^{29}$

For suspensions with an arbitrary size distribution, we must directly deal with Eq. (3), which establishes that $g^{(1)}(q, \tau)$ is the Laplace transform of $G(\Gamma)$. This transformation is a special case of the more general Fredholm integrals of the first kind. Unfortunately, the inversion of this integral is an ill-posed problem and is therefore characterized by the lack of unique solutions and by the high sensitivity of individual solutions to small perturbations in $g^{(1)}(q, \tau)$. To illustrate this, consider that $g^{(1)}(q, \tau)$ and the kernel $\exp (-\Gamma \tau)$ in Eq. (3) are given. We seek to obtain $G(\Gamma)$. Now consider that we perturb the solution in the following way:

$$
\Delta G(\Gamma)=\epsilon \sin (2 n \pi \Gamma), \text { with } n=1,2,3, \ldots,
$$

where $\epsilon$, the perturbation amplitude, indirectly represents the amount of noise in the experiment. The corresponding perturbation in $g^{(1)}(q, \tau)$ will be

$$
\Delta g(q, \tau)=\epsilon \int_{0}^{\infty} d \Gamma \exp (-\Gamma \tau) \sin (2 n \pi \Gamma),
$$

with $n=1,2,3, \ldots$, which, due to the Riemann-Lebesgue lemma, ${ }^{30}$ implies that $\Delta g \rightarrow 0$ as $n \rightarrow \infty .{ }^{31}$ Hence, the ratio $|\Delta G| /|\Delta g|$ becomes arbitrarily large if the perturbation oscillates rapidly enough. As a result, an arbitrarily small perturbation in the experimental data can result in a large change in the computed size distribution. Therefore, the problem is mathematically ill-posed. The CONTIN method attempts to address this issue. One starts by discretizing Eq. (3),

$$
g^{(1)}\left(q, \tau_{m}\right)=\sum_{n=1}^{N} \tilde{G}\left(\Gamma_{n}\right) \exp \left(-\Gamma_{n} \tau_{m}\right),
$$

where $\sum_{n=1}^{N} \tilde{G}\left(\Gamma_{n}\right)=\sum_{n=1}^{N} G\left(\Gamma_{n}\right) \Delta \Gamma_{n}=1$, with $\Delta \Gamma_{n}$ the $\Gamma$ range around point $n$. This we can further rewrite as

$$
g_{m}=A_{m, n} \cdot x_{n}
$$

with $g_{m}$ a vector containing the field correlation data that are associated with a correlation time vector $\tau_{m}, x_{n}=\tilde{G}\left(\Gamma_{n}\right)$ a vector containing the unknown decay rate distribution, and $A_{m, n}$ a transfer matrix representing the kernel of the Laplace transform and defined by $A_{m, n}=\exp \left(-\Gamma_{n} \tau_{m}\right)$. The dimension of the matrix associated with this operator is $N_{y} \times N_{g}$, where $N_{y}$ is the number of components in $g_{m}$, corresponding to the number of points in the experimental correlation function, and $N_{g}$ is the number of components in $x_{n}$, corresponding to the number of points in the computed decay rate distribution.

At first glance, the parameters of the fit can be simply obtained by casting Eq. (3) as a least squares minimization problem,

$$
U=\|A x-g\|^{2} .
$$

However, because this problem is ill-posed, an additional "quality control" side constraint must be introduced. We then cast the problem as

$$
V(\alpha)=\|A x-g\|^{2}+\alpha^{2}\|\omega-\Omega x\|^{2}=\min ,
$$

where $g$ corresponds to the experimental data, $x$ is the recovered decay rate distribution that we seek and $A x$ is the recovered field correlation data. The first term, $\|e\|^{2}=\| A x$ $-g \|^{2}$, is the residual norm, and the second term, $\alpha^{2}\|\omega-\Omega x\|^{2}$, is our side constraint, with $\alpha$ the regularizer. $\omega$ is a term that includes prior knowledge, if any, about the expected solution, while the matrix $\Omega$ constrains the solution in some way. In our case, we do not include any explicit prior knowledge about the solution, and thus $\omega=0$. We take the side constraint to be the curvature of $x$ and, therefore, we choose $\Omega$ as the matrix giving the second derivative of $x$, as given in Ref. 20. However, other choices are possible.

As you can see, the side constraint is related to the solution, which then directly enters into the minimization problem. Note that the relative strength of the regularization depends on the regularizer $\alpha$ : a large $\alpha$ favors a large residual norm at the cost of a small side constraint, while a small $\alpha$ favors a small residual norm at the cost of a large side constraint. This trade-off is common to all regularization methods for computing stable solutions to ill-posed inverse problems. The best-fit parameters will thus not only closely reproduce the experimental data but also will be the most stable to perturbations both in the fit quality and, in our case, with respect to the curvature of the distribution. This stability is optimized by appropriately choosing the regularizer. This is, however, not a trivial issue and is, in fact, the reason why the 
CONTIN method yields results that can dramatically change depending on the selection of $\alpha$.

We note that while our choices of $\omega$ and $\Omega$ do not assume a specific form, for example, a Gaussian, for the distribution, it does favor solutions that are "smooth" in some fashion, as $\Omega x$ is taken as the curvature of the solution. Thus, this selection would not, for example, correctly fit a distribution with multiple peaks. Strictly speaking, some prior knowledge about the system is required to correctly choose the constraint. For example, in the case of a multimodal distribution, the experimental correlation function would typically display one shoulder for each peak in the distribution, provided these are sufficiently far apart - no assumption is necessary, as the correlation function itself would provide this information. However, when the peaks in the multimodal distribution are not sufficiently separated so that the correlation function simply broadens due to the multimodal character of the distribution, the CONTIN method with a side constraint emphasizing the "smoothness" of $G(\Gamma)$ can be taken to provide a good approximation of the actual size distribution.

\section{A. The optimal regularization parameter}

A variety of regularization parameter-choice strategies have been proposed in recent years. These methods can be divided into the following two classes depending on their assumption about the residual norm:

1. Methods based on a priori estimates of $\|e\|^{2}$.

2. Methods that do not require prior knowledge of $\|e\|^{2}$ but that instead seek to extract the necessary information from the experimental data. ${ }^{32}$

Since having an a priori estimate of $x$ implies prior knowledge of the size distribution one intends to determine, the methods in the second category are most desirable; these include the so called generalized cross-validation method, ${ }^{23}$ the quasioptimality criterion, ${ }^{24}$ and the V-curve or L-curve methods. The probability to reject method encoded in the original CONTIN program also falls under this category. ${ }^{20}$ Among them, the first two are very sensitive to noise and do not provide satisfactory results in many cases. ${ }^{33}$ The V-curve method often provides a value of the regularizer that is too large to provide a reasonable size distribution. ${ }^{25}$ As Provencher pointed out in the original paper, the probability to reject method is not always reliable, meaning that in order to properly solve the problem, prior knowledge about the solution must be used, transforming CONTIN into a solution of the first type. In contrast, simulations with the L-curve method seem to provide reasonable results without including constraints based on prior knowledge. ${ }^{34}$ The idea here is to use CONTIN to invert the decay rate distribution for different $\alpha$-values while calculating the norm of the regularization solution, $\|x\|^{2}$, and the residual norm. The log-log plot of these quantities has a characteristic L-shape with a distinct corner separating a nearly vertical and a nearly horizontal branch. The optimal $\alpha$ is the one precisely corresponding to the corner of the curve. This is easy to understand after analyzing what happens along each of the branches of the L-curve. Along the nearly vertical branch, where $\alpha$ is small, variations in the regularizer cause dispro- portionately large changes in $\|x\|^{2}$, which result in large differences in the resultant decay rate distribution. In contrast, along the nearly horizontal part of the curve, where $\alpha$ is large, variations in the regularizer cause large changes in the residual norm $\|A x-g\|^{2}$, resulting in insensitivity to variations in the resultant decay rate distribution. The value of the regularizer at the corner of the L-curve minimizes the combined effect of these two types of changes.

\section{B. Steps of the algorithm}

We start by generating an array of regularizer values, $\alpha_{i}$, with $i=0,1,2,3, \ldots, n$. We then start with $\alpha_{0}$ and assume, for simplicity, an initially flat decay rate distribution $x_{0}$. We then apply the CONTIN algorithm with fixed regularizer to minimize Eq. (12). Along this minimization process, the distribution $G(\Gamma)$ is evolved from the initial $x_{0}$ to $x_{1}$, from $x_{1}$ to $x_{2}$, etc., using the Nelder-Mead method, which is a commonly used nonlinear optimization technique for minimizing an objective function in a multidimensional space. ${ }^{35}$ In each evolution, we calculate $\left|V\left(\alpha_{i}, x_{j-1}\right)-V\left(\alpha_{i}, x_{j}\right)\right|$ until this difference is smaller than $10^{-16}$. At this point, we take $x_{i}$ as the final solution for $\alpha_{i}$. The process is then repeated for each $\alpha_{i}$, as shown in Fig. 1. For each $\alpha_{i}$, we calculate the solution norm and the residual norm and use them to draw the L-curve. We then determine its corner, which further corresponds to the location where the curvature is highest $;^{33}$ this can be done, for example, using the MATLAB program $l_{-}$corner developed by Hansen. ${ }^{33}$ The minimum $V(\alpha)$ for the associated value of $\alpha$ provides the sought after distribution $G(\Gamma)$.

\section{EXPERIMENTAL DETAILS AND METHODS}

DLS experiments were carried out using an LS Instruments 3D DLS spectrometer, which is equipped with a vertically polarized He-Ne laser with wavelength $\lambda_{0}=632.8 \mathrm{~nm}$ as the light source, a motor-driven goniometer, two APD photon detectors, and a two-channel, multiple- $\tau$ digital correlator connected to a personal computer (PC). All measurements were made in water (refractive index $n=1.331$ at $\lambda_{0}$ ) at $21^{\circ} \mathrm{C}$, where the viscosity is $\eta=0.978 \mathrm{cP}$.

The SANS measurements were performed using two small-angle instruments, SANS-I and SANS-II, at the Paul Scherrer Institute, Switzerland. On SANS-I, we used two configurations to access the $q$-range of interest: sampledetector distance $d_{\mathrm{SD}}=18 \mathrm{~m}$, wavelength $\lambda=0.8 \mathrm{~nm}$; and $d_{\mathrm{SD}}=4.5 \mathrm{~m}, \lambda=0.8 \mathrm{~nm}$. On SANS-II, we used the configurations $d_{\mathrm{SD}}=6 \mathrm{~m}, \lambda=1.054 \mathrm{~nm}$, and $d_{\mathrm{SD}}=3 \mathrm{~m}, \lambda=0.53 \mathrm{~nm}$. All experiments were performed at a temperature of (18.0 $\pm 0.5)^{\circ} \mathrm{C}$. Both instruments have a ${ }^{3} \mathrm{He}$ detector with 128 $\times 128$ pixels and pixel areas of $7.5^{2} \mathrm{~mm}^{2}$ and $4.3^{2} \mathrm{~mm}^{2}$ for SANS-I and SANS-II, respectively.

The samples are composed of poly-( $\mathrm{N}$-isopropylacryl amide) (PNIPAM) particles suspended in water. They are synthesized by precipitation polymerization with a total monomer concentration of $100 \mathrm{mM}$. The NIPAM monomer (98 wt. \%), the crosslinker (N,N'-methylenebisacrylamide, 2 wt. \%), and the surfactant sodium dodecyl sulfate (SDS) at a concentration that varies between $0.50 \mathrm{mM}$ and $1.25 \mathrm{mM}$ 


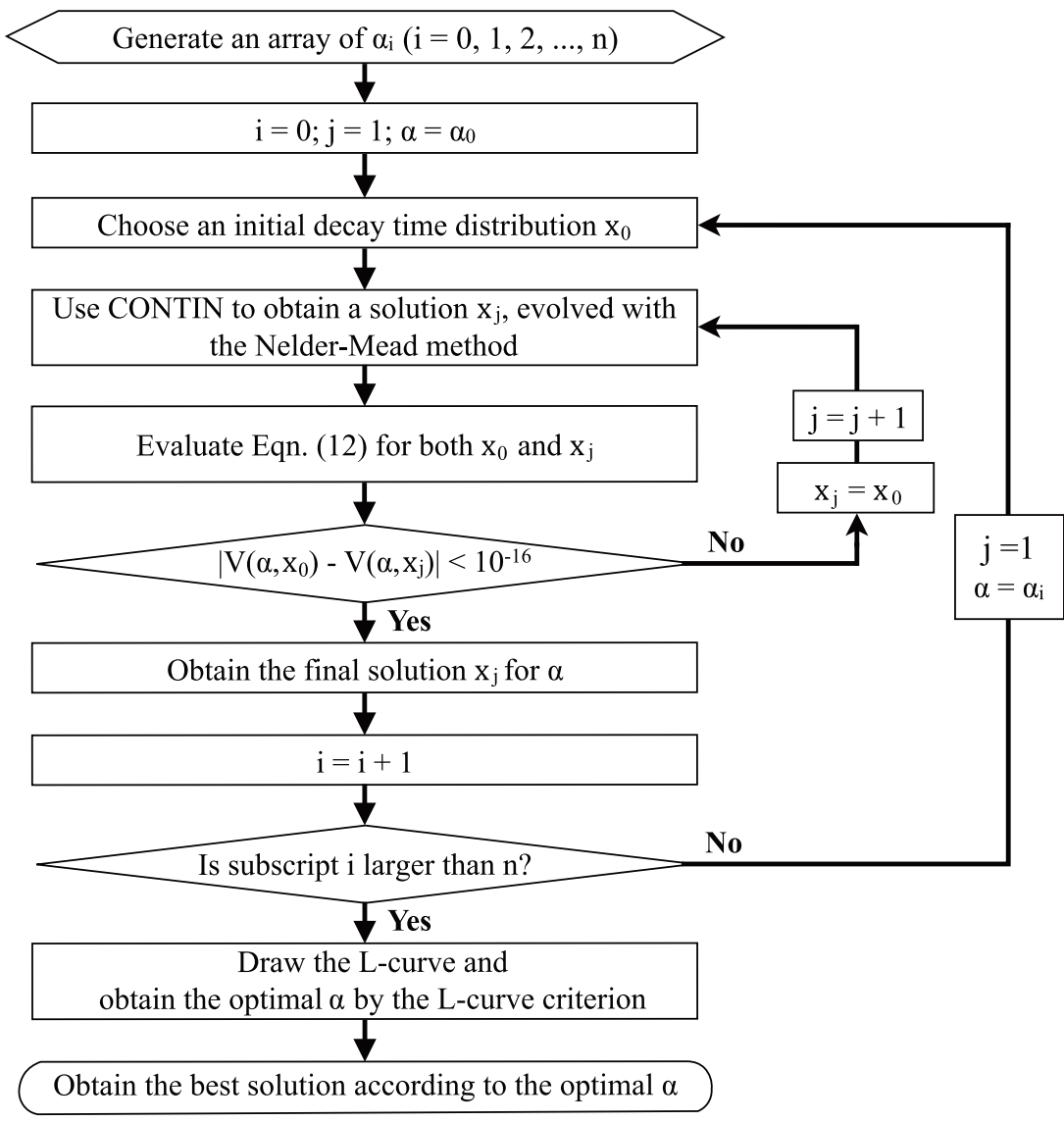

FIG. 1. Flow chart of the CONTIN algorithm incorporating the L-curve criterion. depending on the desired particle size, are all dissolved in $995 \mathrm{ml}$ of distilled and deionized water. The solution is then filtered through a $0.2 \mu \mathrm{m}$ Supor membrane filter and introduced into a 2 liter, 3 -neck round bottom flask. The solution is then heated to $70^{\circ} \mathrm{C}$ by immersing it into a water bath and subjected to a nitrogen atmosphere while stirring. After $1 \mathrm{~h}$, the reaction is initiated by addition of $5 \mathrm{ml}$ of ammonium persulfate (APS, $1.0 \mathrm{mM}$ ) and left for $22 \mathrm{~h}$. The resultant suspension is then cooled down to room temperature, filtered through a $0.8 \mu \mathrm{m}$ Supor membrane filter to remove undesired aggregates, and cleaned using dialysis. We then freeze-dry the suspension and redisperse it in either water or deuterated water for use in the light or neutron scattering experiments, respectively. We refer to samples made in this way as s-samples.

We also mix s-samples together to obtain additional suspensions. We refer to these suspensions as m-samples.

\section{RESULTS AND DISCUSSION}

The field correlation functions we obtain for all our dilute samples exhibit a single decay due to particle diffusion, as shown by the points in Fig. 2(a) for sample s1. To obtain the size distribution, we apply the CONTIN algorithm together with the L-curve criterion for selecting the regularizer. We start by choosing an $\alpha$ value of $10^{-4}$ and a flat distribution of decay rates, $x_{0}$, shown with a dashed line in Fig. 2(b). With this initial information, we minimize $V\left(\alpha_{0}\right)$ by applying the CONTIN algorithm with fixed regularizer. In this process, the decay rate distribution is progressively evolved with the Nelder-Mead method, as shown for the first three evolutions also in Fig. 2(b). Once the minimum in $V\left(\alpha_{0}\right)$ is obtained, we calculate $\|x\|^{2}$ and $\|A x-g\|^{2}$ using the corresponding decay rate distribution and plot them against each other, as shown in Fig. 2(c). For this first regularizer value, we generate the leftmost point in this plot.

We then choose a second value for the regularizer, and repeat the procedure above to obtain a second point in the plot of $\|x\|^{2}$ versus $\|A x-g\|^{2}$. This is continued until $\alpha=10^{2}$. The resulting curve is L-shaped, as shown in Fig. 2(c). The optimal regularizer corresponds to the absolute minimum of $V(\alpha)$. For sample $\mathrm{s} 1$, this corresponds to $\alpha=0.0053$, as shown in Fig. 2(d). This minimum is identified as the corner of the L-curve.

Using this regularizer, we apply the CONTIN algorithm once again to obtain the final decay rate distribution, shown in Fig. 2(e). For sample s1, we obtain $\bar{\Gamma}=702.3 \mathrm{~Hz}, \sqrt{k_{2}}$ $=84.2 \mathrm{~Hz}$, and a relative polydispersity $\sqrt{k_{2} / \bar{\Gamma}^{2}} \times 100 \%$ $=12.0 \%$. The $g^{(1)}(q, \tau)$ associated with this final decay rate distribution is shown with a line in Fig. 2(a).

To obtain the size distribution, we change variables in Eq. (3) using $\Gamma=\left(k_{B} T q^{2}\right) /\left(6 \pi \eta R_{h}\right)=A / R_{h}$. The result is

$$
g^{(1)}(q, \tau)=\int_{0}^{\infty} F\left(R_{h}\right) \exp \left(-A \tau / R_{h}\right) d R_{h},
$$

where $F\left(R_{h}\right)=\left(A / R_{h}^{2}\right) G(\Gamma)$ is the size distribution, which is also normalized so that $\int_{0}^{\infty} F\left(R_{h}\right) d R_{h}=1$. For sample s1, the result is shown in Fig. 2(f). We obtain a mean radius $\bar{R}_{h}$ $=\int_{0}^{\infty} F\left(R_{h}\right) R_{h} d R_{h}=(135 \pm 7) \mathrm{nm}$ and a square root variance $\sigma_{R}=\sqrt{\int_{0}^{\infty} F\left(R_{h}\right)\left(R_{h}-\bar{R}_{h}\right)^{2} d R_{h}}=14.85 \mathrm{~nm}$; the associated 

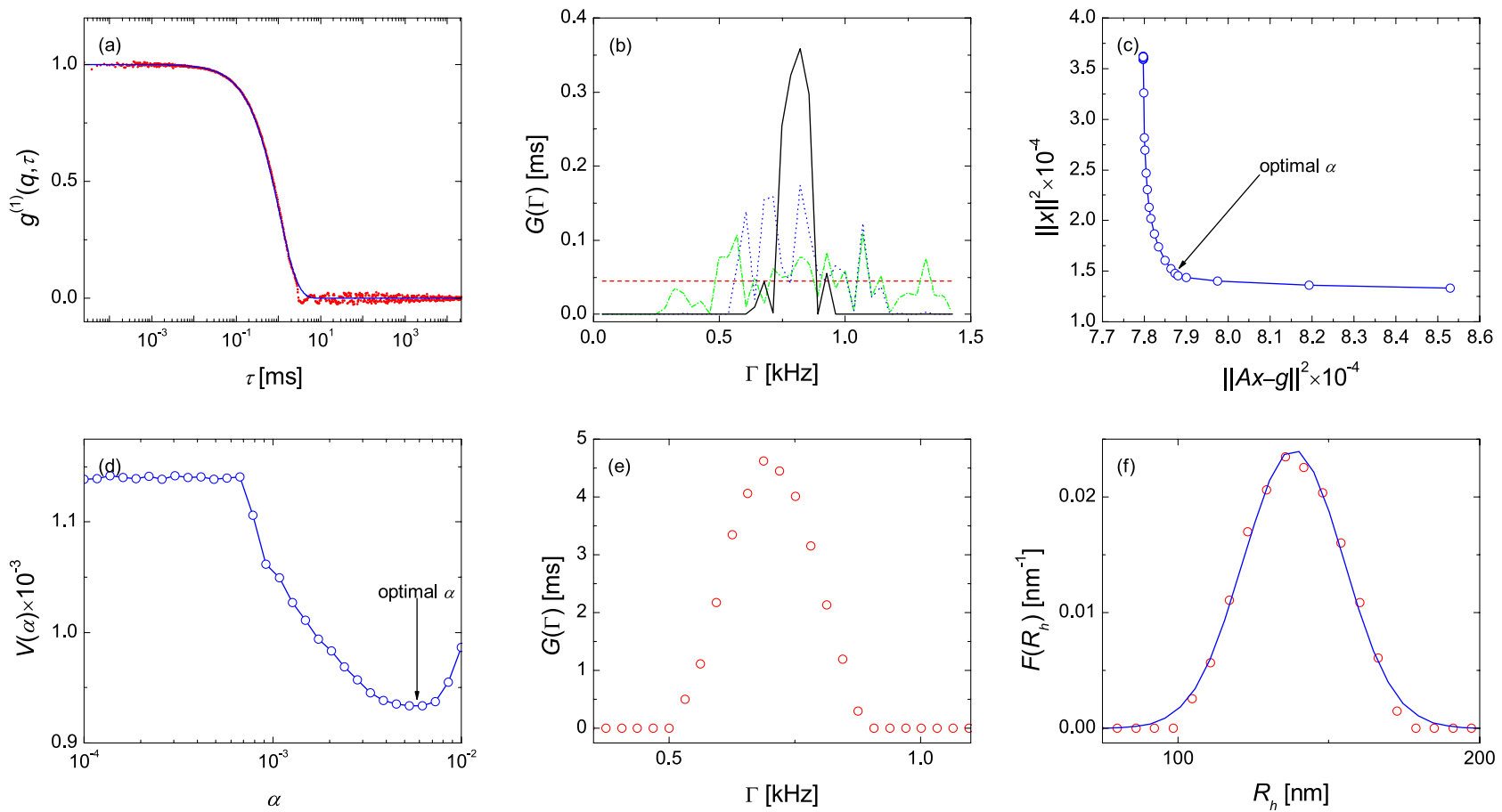

FIG. 2. (a) Field correlation function for sample s1 (red circles) and final CONTIN fit (solid line). (b) Initial decay rate distribution, $x_{0}$ (red dashed line), and three subsequent evolutions: $x_{1}$ (green dashed-dotted line), $x_{2}$ (blue dotted line), and $x_{3}$ (black solid line). (c) L-curve. (d) Minimizing function $V(\alpha)$ versus $\alpha$. (e) Final decay rate distribution. (f) Particle hydrodynamic radius distribution (red circles) and a Gaussian fit to the data (blue curve); the fit yields $R_{h}=(138.3 \pm 0.7) \mathrm{nm}, \sigma_{R}=(16.6 \pm 0.3) \mathrm{nm}$, and $p=(12.0 \% \pm 0.5 \%)$. Light scattering data were taken at $\theta=135^{\circ}$ for $t=150 \mathrm{~s}$. The CONTIN analysis was carried out with $N_{g}=160$ over an integration range of $[1,500] \mathrm{nm}$.

relative polydispersity is $p=\sqrt{\sigma_{R}^{2} / \bar{R}_{h}^{2}} \times 100 \%=(11 \pm 3) \%$, as shown in Table I. We emphasize that $\bar{R}_{h}$ is the mean and $\sigma_{R}$ the standard deviation of the hydrodynamic radius distribution function $F\left(R_{h}\right)$. The mean and standard deviation of the corresponding Gaussian fit to $F\left(R_{h}\right)$ give similar values: $R_{h}=(138.3 \pm 0.7) \mathrm{nm}$ and $\sigma_{R}=(16.6 \pm 0.3) \mathrm{nm}$. Note that $p$ is expected to be equal to $\sqrt{k_{2} / \bar{\Gamma}^{2}}, 29$ consistent with our results. We also note that $q R_{h} \lesssim 1 / 2$ over the entire available $q$-range of the light scattering apparatus, therefore allowing us to use the discretization method outlined for Rayleigh scatterers. For the more general case, we refer to the Appendix.

As a check for these results, we first analyze the data using the cumulants method. We fit the field correlation function to a line up to the value of $\tau$ where it has decayed to $10 \%$ of the original amplitude, ${ }^{36}$ as shown in Fig. 3(a) for sample s1.

TABLE I. Summary of the results obtained by inverting the experimental data of s-samples according to the CONTIN algorithm together with the L-curve criterion. For comparison, the results from the cumulant method and SANS measurements are given in the second and third columns, respectively.

\begin{tabular}{lrrrcc}
\hline \hline & \multicolumn{2}{c}{ CONTIN } & \multirow{2}{*}{ Cumulants } & \multicolumn{2}{c}{ SANS } \\
\cline { 2 - 3 } \cline { 5 - 6 } No. & $\bar{R}_{\boldsymbol{h}}(\mathrm{nm})$ & $p(\%)$ & $\bar{R}_{\boldsymbol{h}}(\mathrm{nm})$ & $R_{\text {SANS }}(\mathrm{nm})$ & $\sigma_{\text {poly }}(\%)$ \\
\hline s1 & $135 \pm 7$ & $11 \pm 3$ & $139 \pm 1$ & $133.2 \pm 0.6$ & $10.0 \pm 2.0$ \\
s2 & $81 \pm 4$ & $17 \pm 4$ & $78.6 \pm 0.6$ & $71 \pm 1$ & $23 \pm 5$ \\
s3 & $101 \pm 5$ & $14 \pm 4$ & $97.0 \pm 0.9$ & $93 \pm 3$ & $15 \pm 3$ \\
s4 & $142 \pm 7$ & $11 \pm 3$ & $144 \pm 1$ & $137.5 \pm 0.6$ & $10.2 \pm 2.0$ \\
s5 & $173 \pm 9$ & $13 \pm 3$ & $166 \pm 1$ & $159 \pm 2$ & $11 \pm 2.3$ \\
s6 & $130 \pm 7$ & $10 \pm 3$ & $128 \pm 0.5$ & $126 \pm 1$ & $10.8 \pm 2.2$ \\
\hline \hline
\end{tabular}

We obtain $\bar{R}_{h}=(139 \pm 1) \mathrm{nm}$, consistent with the mean size obtained from the CONTIN analysis, as shown in Table I.

We also perform SANS as an alternative route to access the mean particle radius and the suspension polydispersity. This is done with dilute samples such that interactions between the particles are not important. In these conditions, the structure factor $S(q) \approx 1$ and therefore we directly determine the particle form factor $P(q)$. We find that $P(q)$ monotonously decreases with $q$, as shown in Fig. 3(b) for sample s1, consistent with previous measurements with similar microgels. ${ }^{37-40}$

To analyze the SANS data, we use a core-shell model. ${ }^{41}$ We model our microgel particles as consisting of a dense, spherical core of radius $R$, with a constant scattering length density. In $q$-space, this contribution is the form factor of a homogeneous sphere,

$$
P_{\mathrm{hom}(q)}=\left(\frac{3[\sin (q R)-q R \cos (q R)]}{(q R)^{3}}\right)^{2} .
$$

The shell part accounts for a decaying cross-linker concentration from the particle center to its periphery. ${ }^{42}$ Hence, the particle core is surrounded with an inhomogeneous, fuzzy shell of polymer. This fuzziness is accounted for by convoluting the core with a Gaussian function of a second length scale $\sigma_{\text {surf }}$, which is related to the shell thickness

$$
P_{\text {inho }}(q)=\left[\frac{3[\sin (q R)-q R \cos (q R)]}{(q R)^{3}} \exp \left(-\frac{\left(\sigma_{\text {surf }} q\right)^{2}}{2}\right)\right]^{2} .
$$

The overall radius of the particles is $R_{\text {SANS }}=R+2 \sigma_{\text {surf }}$; this is expected to be smaller than $R_{h}$ determined by DLS because SANS is less sensitive to the dangling polymer chains 

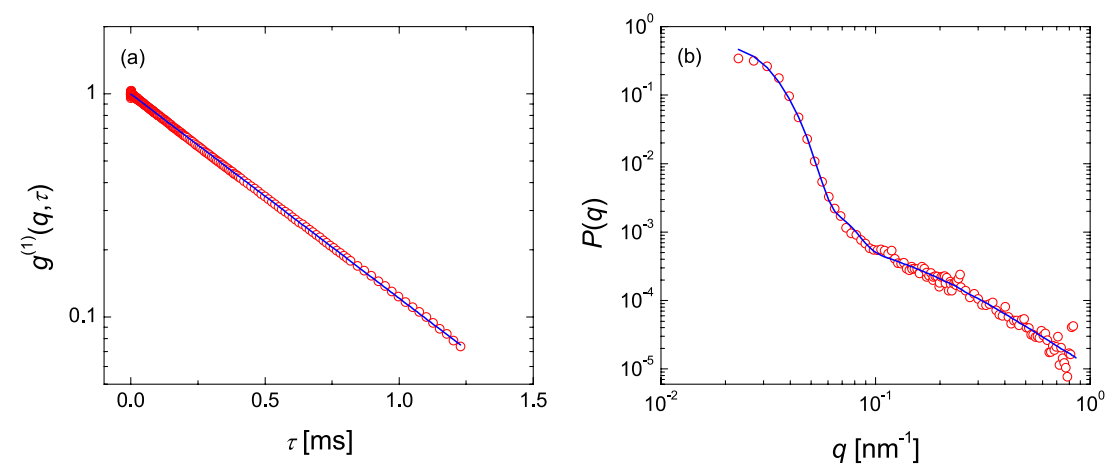

FIG. 3. (a) Logarithm of the field correlation function versus the correlation time together with a cumulant fit to linear order. (b) Form factor obtained with SANS and the corresponding fit with Eq. (18). The data are for sample s1.

at the particle periphery. We further consider polydispersity in the core size according to a Gaussian distribution,

$$
D\left(R, \bar{R}, \sigma_{\text {poly }}\right)=\frac{1}{\sqrt{2 \pi} \sigma_{\text {poly }} \bar{R}} \exp \left(-\frac{(R-\bar{R})^{2}}{2 \sigma_{\text {poly }}^{2} \bar{R}^{2}}\right),
$$

where $\sigma_{\text {poly }}$ is the associated relative polydispersity of the core. To complete our model, we add contributions to the form factor that take into account the internal structure of the microgel particles. We use a Lorentzian function,

$$
I_{\text {fluct }}(q)=\frac{I_{\text {fluct }}(0)}{1+\xi^{2} q^{2}},
$$

where $I_{\text {fluct }}(0)$ is the intensity at $q=0$ and $\xi$ represents the characteristic mesh size of the intraparticle polymer network. We also add a constant contribution to the scattered intensity, $B G$, to account for contributions due to incoherent scattering. Finally, we account for the smearing of the data by the instrument. ${ }^{43,44}$ In $q$-space, this corresponds to multiplying our model with a Gaussian, $H\left(q^{\prime}, q, \sigma_{\text {smear }}\right)$, with $\sigma_{\text {smear }}$ the instrumental smearing. Our final model is then written as

$$
\begin{aligned}
P(q)= & \frac{1}{\left\langle V^{2}\right\rangle} \int_{0}^{\infty} d R D\left(R, \bar{R}, \sigma_{\text {poly }}\right) V^{2}(R) \\
& \times \int_{0}^{\infty} d q^{\prime}\left[P_{\text {inho }}\left(q^{\prime}\right)+I_{\text {fluct }}\left(q^{\prime}\right)\right] \\
& \times H\left(q^{\prime}, q, \sigma_{\text {smear }}\right)+B G,
\end{aligned}
$$

where $H\left(q^{\prime}, q, \sigma_{\text {smear }}\right)=\frac{1}{\sqrt{2 \pi} \sigma_{\text {smear }}} \exp \left[-\frac{\left(q^{\prime}-q\right)^{2}}{2 \sigma_{\text {smear }}^{2}}\right]$ and $\left\langle V^{2}\right\rangle$ $=\left[\int_{0}^{\infty} d R V^{2}(R) D\left(R, \bar{R}, \sigma_{\text {poly }}\right)\right] /\left[\sqrt{2 \pi} \sigma_{\text {poly }} \bar{R}\right]$ with $V(R)=4 \pi$ $R^{3} / 3$ the volume of the core. By fitting the data obtained for sample s1 to this model, leaving $R, \sigma_{\text {surf }}, \sigma_{\text {poly }}, \xi, I_{\text {fluct }}(0)$, and
$B G$ as fitting parameters, we obtain $R_{\mathrm{SANS}}=(133.2 \pm 0.6) \mathrm{nm}$ and a relative polydispersity for the core $\sigma_{\text {poly }}=(10.0$ $\pm 2.0) \%$, as shown in Table I. These values agree well with those obtained using the CONTIN algorithm considering that $R_{S A N S}$ is expected to be lower than the hydrodynamic radius as mentioned above.

Equally satisfactory is the agreement obtained for all other s-samples, as also shown in Table I; these span a range in hydrodynamic radius from $\sim 81 \mathrm{~nm}$ to $\sim 173 \mathrm{~nm}$ and a relative polydispersity range from $\sim 10 \%$ to $\sim 17 \%$.

To further test the CONTIN method with the L-curve criterion for choosing the regularizer, we prepare three suspensions obtained from mixtures of the s-samples. The results for the mean particle radius and the relative polydispersity obtained from the fit of the SANS data to the form factor model, shown in Fig. 4(a) for sample m3, and of the mean radius obtained from the cumulant analysis, shown in Fig. 4(b) for the same sample, are shown in Table II. The corresponding results from the CONTIN analysis yield size distributions like that shown in Fig. 4(c) for sample m3, which agree with the size distributions that result from the neutron scattering analysis, and mean size and polydispersities that are also consistent with the cumulants and neutron scattering results (see Table II). Hence, the CONTIN algorithm together with the L-curve criterion for selecting the regularizer of the regularization process correctly accounts for the size distribution of the microgel suspensions measured by independent means.

We realize, however, that the results for sample $\mathrm{m} 1$ are not satisfactory. This can be traced back to the fact that the size distribution expected from the proportion of s-samples used in this mixture exhibits peaks associated to the sizes
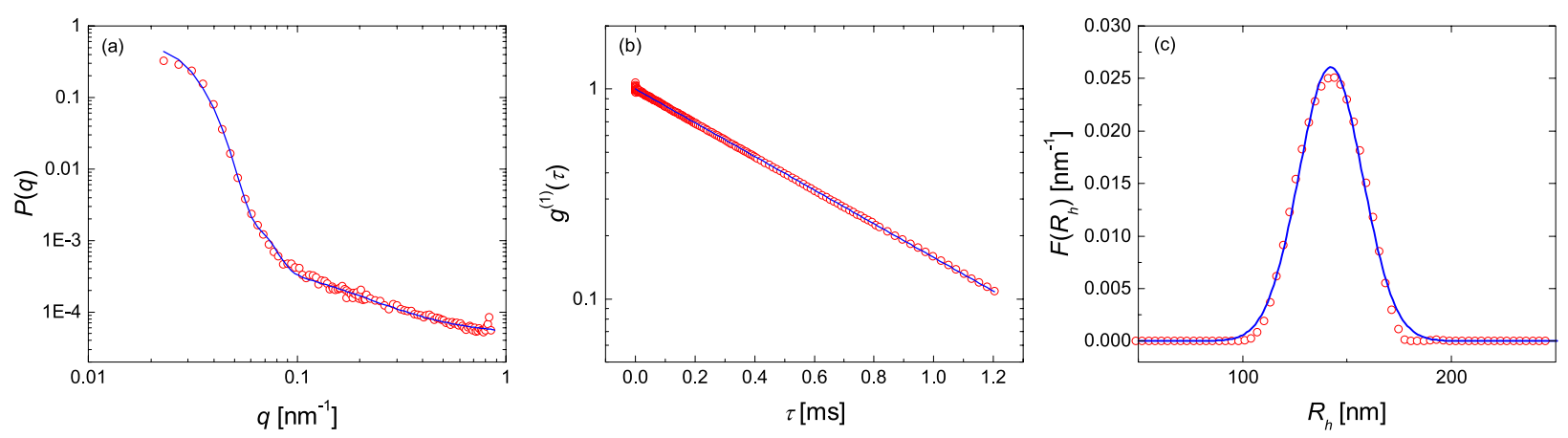

FIG. 4. (a) SANS form factor and associated fit. (b) Field correlation function versus the correlation time together with a first-order cumulant fit. (c) Particle size distribution obtained from the CONTIN analysis together with the L-curve criterion. The line is a Gaussian fit to the data; the fit yields $R_{h}=(142.3 \pm 0.1) \mathrm{nm}$, $\sigma_{R}=(15.3 \pm 0.1) \mathrm{nm}$, and $p=(10.8 \% \pm 0.1 \%)$. All panels refer to sample m3. Light scattering data were taken at $\theta=120^{\circ}$ for $t=150 \mathrm{~s}$. The CONTIN analysis was carried out with $N_{g}=160$ over an integration range of $[1,500] \mathrm{nm}$. 
TABLE II. Summary of the results obtained for the m-samples with the CONTIN algorithm together with the L-curve criterion, the cumulant method, and the complementary SANS measurements. The m-samples are obtained from mixing s-samples. The weight fraction of each of the s-samples is given in parentheses in the second column of the table. The radii are given in units of $\mathrm{nm}$.

\begin{tabular}{|c|c|c|c|c|c|c|}
\hline & \multirow{2}{*}{$\begin{array}{c}\text { s-samples } \\
(\%)\end{array}$} & \multicolumn{2}{|c|}{ CONTIN } & \multirow{2}{*}{$\begin{array}{c}\text { Cumulants } \\
\qquad \bar{R}_{h}\end{array}$} & \multicolumn{2}{|c|}{ SANS } \\
\hline & & $\bar{R}_{h}$ & $p(\%)$ & & $R_{\text {SANS }}$ & $\sigma_{\text {poly }}(\%)$ \\
\hline $\mathrm{m} 1$ & $\begin{array}{l}\text { s1(23) } \\
\text { s2(1) } \\
\text { s3(15) } \\
\text { s5(29) } \\
\text { s6(32) }\end{array}$ & $140 \pm 6$ & $13 \pm 4$ & $141 \pm 5$ & $120 \pm 2$ & $22 \pm 4$ \\
\hline $\mathrm{m} 2$ & $\begin{array}{c}\mathrm{s} 1(40) \\
\mathrm{s} 3(8.5) \\
\mathrm{s} 5(23.5) \\
\mathrm{s} 6(28)\end{array}$ & $138 \pm 7$ & $14 \pm 4$ & $139 \pm 5$ & $125 \pm 4$ & $14 \pm 3$ \\
\hline $\mathrm{m} 3$ & $\begin{array}{l}s 4(66) \\
s 6(34)\end{array}$ & $142 \pm 7$ & $11 \pm 3$ & $147 \pm 6$ & $131.7 \pm 0.9$ & $10.5 \pm 2.1$ \\
\hline
\end{tabular}

of the s-samples and is thus not as well described by a Gaussian function. Since our selection of side constraint favors distributions with smaller curvature, the CONTIN results are expected to be less accurate for this sample than for the rest.

\section{L-CURVE VS. PROBABILITY TO REJECT}

\section{A. The probability to reject method}

In the probability to reject method, the idea is to perform an $F$-test with the regularized solution, $x$, for different values of $\alpha$, with respect to the solution obtained with the lowest value of $\alpha$. The fractional increase of $V(\alpha)$, also referred to as the $F$-statistic, is defined as

$$
F(\alpha)=\frac{V(\alpha)-V\left(\alpha_{0}\right)}{V\left(\alpha_{0}\right)} \frac{N_{y}-N_{g}}{N_{g}} .
$$

In this equation, $V\left(\alpha_{0}\right)$ is the value of $V(\alpha)$ for the smallest $\alpha$ used; in our case, this value is $\alpha_{0}=10^{-4}$. We thus use $V\left(\alpha_{0}\right)$ as a reference because this is the value of $V(\alpha)$ that
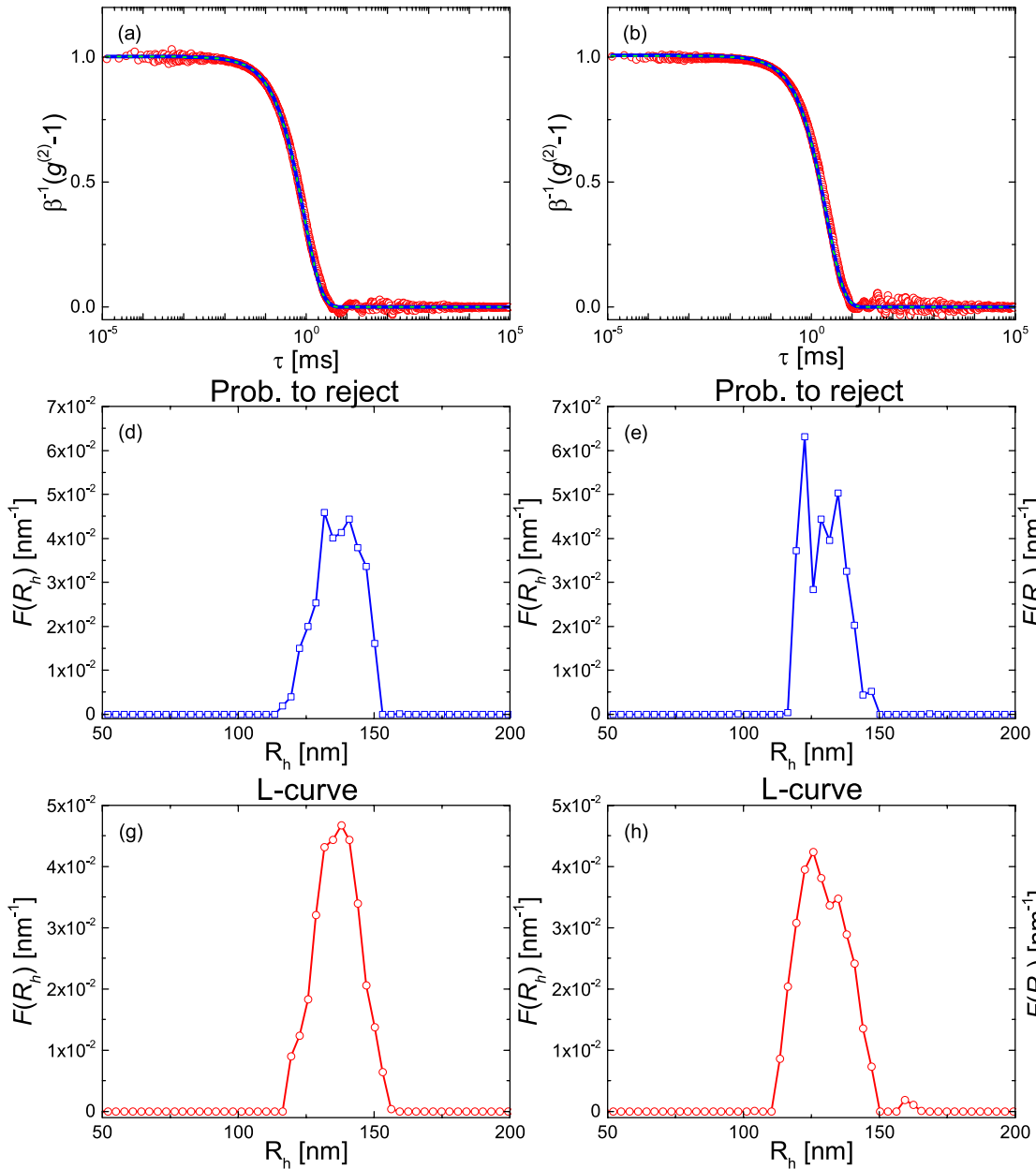

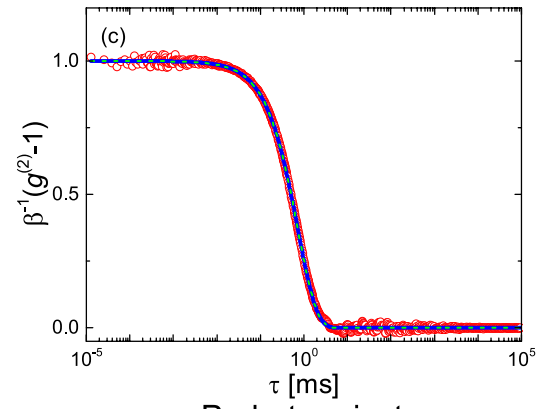

Prob. to reject
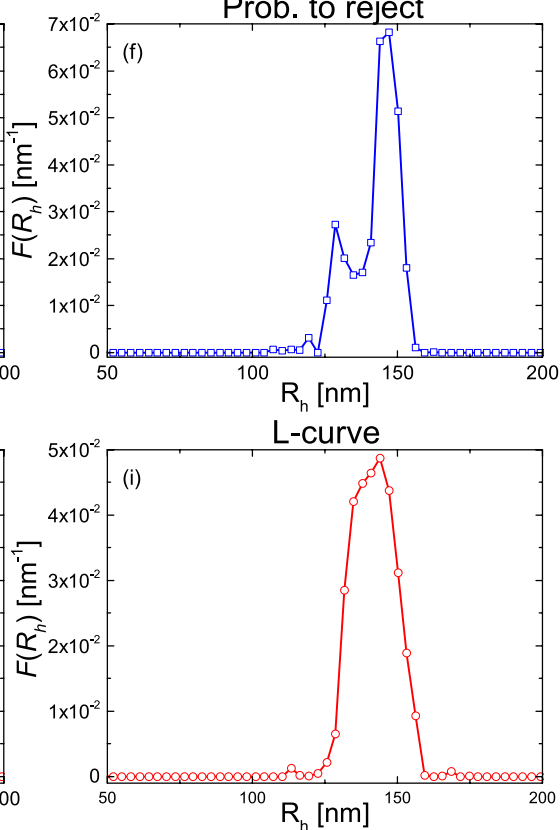

FIG. 5. Data analysis of sample s 1 at (a), (d), (g) $\theta=90^{\circ}$, (b), (e), (h) $\theta=50^{\circ}$, and (c), (f), (i) $\theta=105^{\circ}$. (a) $-(\mathrm{c})$ Experimental correlation functions (red circles) and data fits according to an $\alpha$ determined with L-curve (blue line) and the probability to reject (green dashed line) criteria. (d)-(f) Size distributions computed with the best $\alpha$ obtained with the probability to reject criterion. (g)-(i) Size distributions computed with the best $\alpha$ obtained with the L-curve criterion. These data sets were acquired over a measurement time of $150 \mathrm{~s}$. For the analysis, we use $N_{g}=160$ and the range of integration is [1, 500] nm. 
corresponds to the decay rate distribution computed with the smallest $\alpha$, where the effect of the regularizer is weakest. The term $\left[V(\alpha)-V\left(\alpha_{0}\right)\right] / V\left(\alpha_{0}\right)$ therefore quantifies the effect of the side constraint, which in our case is related to the smoothness of the decay rate distribution. The quantity $F(\alpha)$ is $F$-distributed and thus has an associated probability distribution function given by

$$
\begin{aligned}
f\left(F(\alpha) \mid N_{g}, N_{y}-N_{g}\right)= & \frac{\left(\frac{N_{g}}{N_{y}-N_{g}}\right)^{\frac{N_{g}}{2}}}{B\left(\frac{N_{g}}{2}, \frac{N_{y}-N_{g}}{2}\right)} \times F(\alpha)^{N_{g} / 2-1} \\
& \times\left(1+\frac{N_{g}}{N_{y}-N_{g}} F(\alpha)\right)^{-N_{y} / 2},
\end{aligned}
$$

where $B(x, y)$ is the beta function. The selection of $\alpha$ then follows from choosing a value for the cumulative distribution of $f\left(F(\alpha) \mid N_{g}, N_{y}-N_{g}\right)$, defined as

$$
\begin{aligned}
f_{c}\left(F(\alpha) \mid N_{g}, N_{y}-N_{g}\right) & \\
\quad= & \int_{0}^{F(\alpha)} f\left(X \mid N_{g}, N_{y}-N_{g}\right) d X \\
& =B\left(\frac{N_{g} F(\alpha)}{N_{g}[F(\alpha)-1]+N_{y}} \mid \frac{N_{g}}{2}, \frac{N_{y}-N_{g}}{2}\right),
\end{aligned}
$$

where $B(z \mid x, y)$ is the regularized incomplete beta function. The basic idea to select a specific value of $f_{c}\left(F(\alpha) \mid N_{g}, N_{y}\right.$ $\left.-N_{g}\right)$ is to compare solutions with different $\alpha$ and thus different weights for the smoothness in Eq. (12) and determine how the $F$-statistic is affected by the regularization process. For values of $f_{c}\left(F(\alpha) \mid N_{g}, N_{y}-N_{g}\right)$ close to its maximum value of 1 , the change in the regularizer has a large effect on the solution. In this regime, the regularizer is considered to have a too-strong effect on the computed solution, leading to an over-smoothed decay rate distribution. On the contrary, when $f_{c}\left(F(\alpha) \mid N_{g}, N_{y}-N_{g}\right)$ is close to its minimum value of 0 , the regularizer only weakly affects the solution. In this regime, the regularizer is assumed to have a negligible effect; indeed, for $\alpha=\alpha_{0}$, the problem is essentially an ordinary, unregularized least-squares problem. As a result, the intermediate value of 0.5 is chosen for $f_{c}\left(F(\alpha) \mid N_{g}, N_{y}-N_{g}\right)$ to select the "best $\alpha$ ". This constitutes the "probability to reject" method, ${ }^{20,45,46}$ wherein the selected $\alpha$ is assumed to show the best balance between the goodness of the fit and the smoothness of the computed decay rate distribution.

Provencher, ${ }^{20}$ though, highlights that this criterion is a "rough guide" since (i) $N_{g}$ is to a large extent arbitrary, (ii) the use of the described $F$-test is one of many possible, and
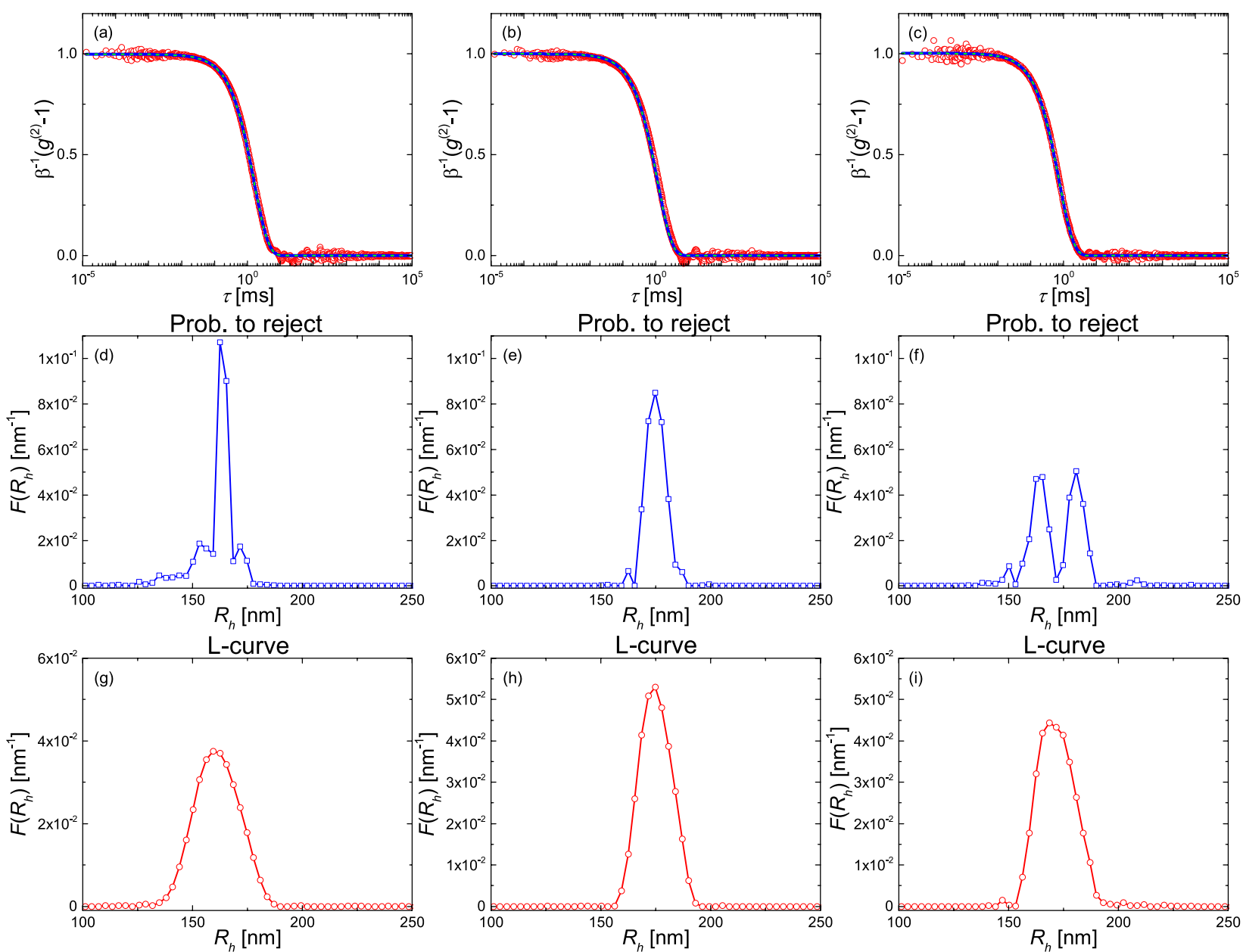

FIG. 6. Data analysis of sample s5 at (a), (d), (g) $\theta=70^{\circ}$, (b), (e), (h) $\theta=90^{\circ}$, and (c), (f), (i) $\theta=120^{\circ}$. (a) $-(\mathrm{c})$ Experimental correlation functions (red circles) and data fits according to an $\alpha$ determined with the L-curve (blue line) and the probability to reject (green dashed line) criteria. (d)-(f) Size distributions computed with the best $\alpha$ obtained with the probability to reject criterion. (g)-(i) Size distributions computed with the best $\alpha$ obtained with the L-curve criterion. These data sets were acquired over a measurement time of $150 \mathrm{~s}$. For the analysis, we use $N_{g}=160$ and a range of integration of [1, 500] nm. 
(iii) the selection of the "best $\alpha$ " depends on the computed decay rate distribution obtained with $\alpha=\alpha_{0}$ through $V\left(\alpha_{0}\right){ }^{45}$ It has also been shown that the probability to reject leads to unsatisfactory results when either the baseline of the data is not well defined or when it possesses some amount of non-random noise. $^{46}$

Our own data support this conclusion. In Figs. 5 and 6, we report the comparison between the size distributions obtained with the $\alpha$-selection using the probability to reject method and the L-curve criterion. We see that the regularizer selected with the probability to reject method leads to distributions that are not as reproducible over several measurements at different scattering angles; we show this for samples s1 and s5 as representative examples. This means that, for our system, the $\alpha$ obtained with the probability to reject method does not give a solution with a good balance between the smoothness of the distribution and the goodness of the fit. Furthermore, the presence of multiple peaks reveals some bias due to the regularization process; the smoothness of the size distribution is not well weighted and, as a consequence, the probability to reject does not select the best $\alpha$. In contrast, the L-curve method leads to more robust results and reliable size distributions.

In addition, we note that the L-curve method gives a more direct comparison between the two quantities that are in competition during the minimization process: the smoothness of the solution, taken into account by the squared norm of the computed solution, $\|x\|^{2}$, and the goodness of the fit, $\|A x-g\|^{2}$. The smoothness is taken into account in the side constraint during the minimization process. The L-curve, particularly the $\|x\|^{2}$ axis, simply reflects that one does not want to choose an $\alpha$, and thus a weight for the side constraint, which is where the smoothness of the decay rate distribution is accounted for, such that the obtained solution changes massively with small changes in the weight of the side constraint. Hence, the smoothness does not enter in $\|x\|^{2}$ per se but it enters in the minimization problem in the side constraint, where it is weighted by $\alpha$. This ensures that we obtain a robust solution.
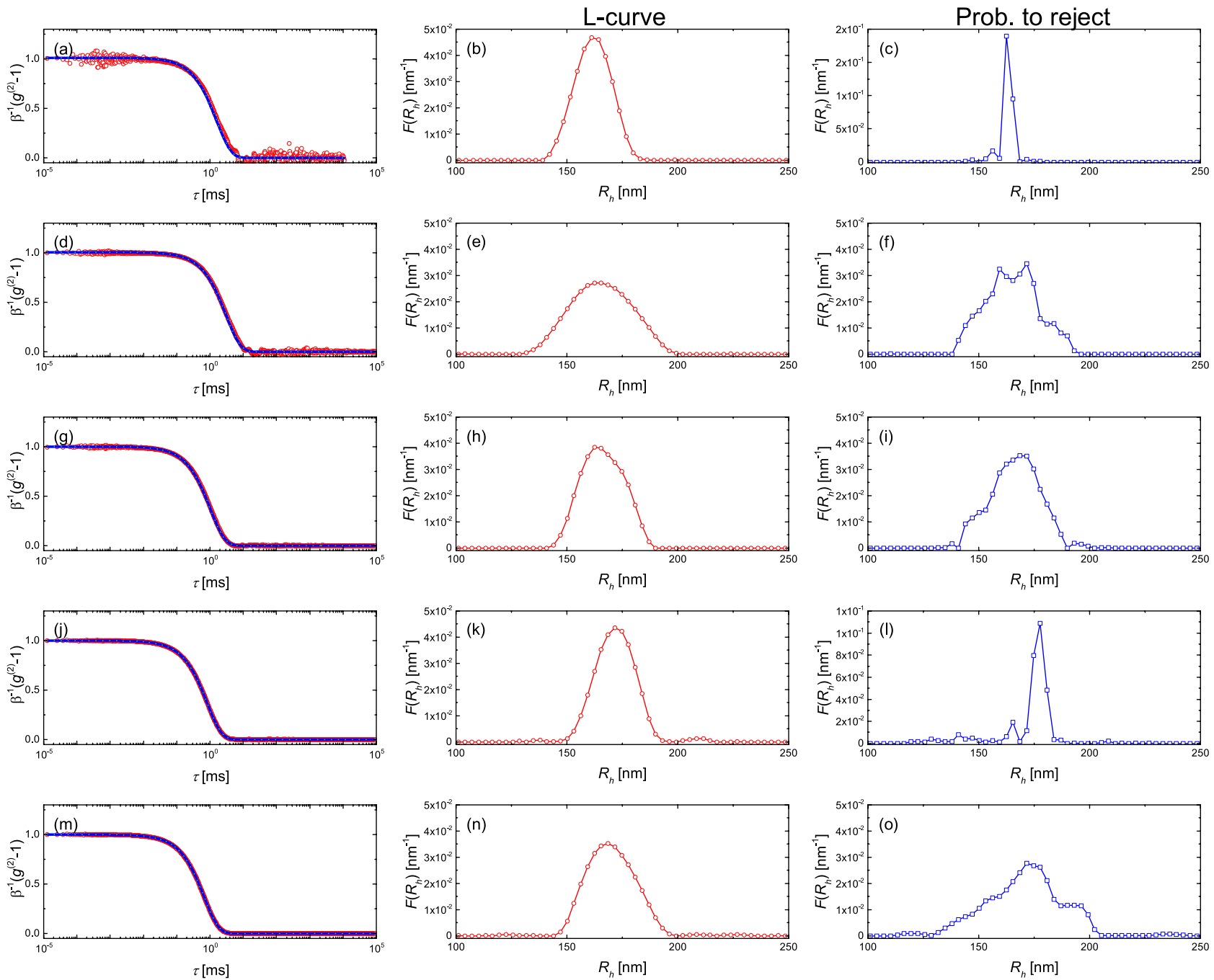

FIG. 7. Data analysis of sample s5 at different angles and acquisition times: (a)-(c) $\theta=70^{\circ}, t=15 \mathrm{~s}$; (d)-(f) $\theta=50^{\circ}, t=150 \mathrm{~s}$; (g)-(i) $\theta=90^{\circ}, t=1500 \mathrm{~s}$; (j)-(l) $\theta=110^{\circ}, t=6000 \mathrm{~s}$; and (m)-(o) $\theta=130^{\circ}, t=60000 \mathrm{~s}$. (a), (d), (g), (j), (m) Experimental correlation functions (red circles) and data fits according to an $\alpha$ determined with L-curve (blue line) and probability to reject (green dashed line) criteria. (b), (e), (h), (k), (n) Size distributions computed with the best $\alpha$ obtained with the L-curve criterion. (c), (f), (i), (l), (o) Size distributions computed with the best $\alpha$ obtained with the probability to reject criterion. For the analysis, we use $N_{g}=160$ and an integration range of $[1,500] \mathrm{nm}$. Note that panels (c), (f), (i), (l), and (o) have been plotted with significantly different y-axes due to the high variability in results obtained with the probability to reject method. 


\section{B. The influence of the measuring time}

Noise in the data is the main issue with an ill-posed problem such as the Laplace inversion under consideration. Minimal sensitivity to noise is desirable in the method for choosing the value of the regularizer. All of the previously shown light scattering measurements have a measurement duration of $150 \mathrm{~s}$. We have tested the L-curve and probability to reject criteria with data acquired with measurement times of $15 \mathrm{~s}, 150 \mathrm{~s}, 1500 \mathrm{~s}, 6000 \mathrm{~s}$, and $60000 \mathrm{~s}$, which correspondingly have different levels of noise in the data. We exemplify the results with representative data for sample s5 and show in Fig. 7 both the resulting fits of $g^{(1)}(q, \tau)$ and the obtained size distributions using both the L-curve and probability to reject criterion. In the left column, decreasing noise with increasing measurement time is clearly visible. The central column shows that the L-curve test returns distributions with consistent means, widths, and overall shape, regardless of the noise level. However, as shown in the right column, the probability to reject method fails the test of reproducibility, displaying not only different widths but also qualitatively different shapes. In the case of the probability to reject method there is also no apparent correlation between the measurement time and the resulting distribution.

\section{The influence of the decay rate range}

The result of the inversion process is a decay rate distribution that we convert into a size distribution (see Eqs. (3) and (13)). If the allowed size range does not extend sufficiently far to both sides of the actual size distribution, the result might exhibit significant deviations. We have thus tested for the possibility that the computed distribution is sensitive to the allowed range in $\Gamma$ and thus in $R_{h}$, comparing the L-curve and probability to reject criterion. As exemplified in Fig. 8 for sample s5, we observe large inconsistencies between different analyses using the probability to reject method, but relatively consistent results from the L-curve method, even when the range is varied by more than an order of magnitude. Note that the points in Figs. 8(f) and 8(i) are logarithmically spaced due to the large $R$-range considered.

\section{The influence of $\boldsymbol{N}_{\boldsymbol{g}}$}

As discussed previously, the number of points allowed in the computed distribution, $N_{g}$, is another parameter in the analysis. We have tested the sensitivity of the L-curve method to different values of $N_{g}$. For an integration range corresponding to sizes between 1 and $500 \mathrm{~nm}$, we have tested
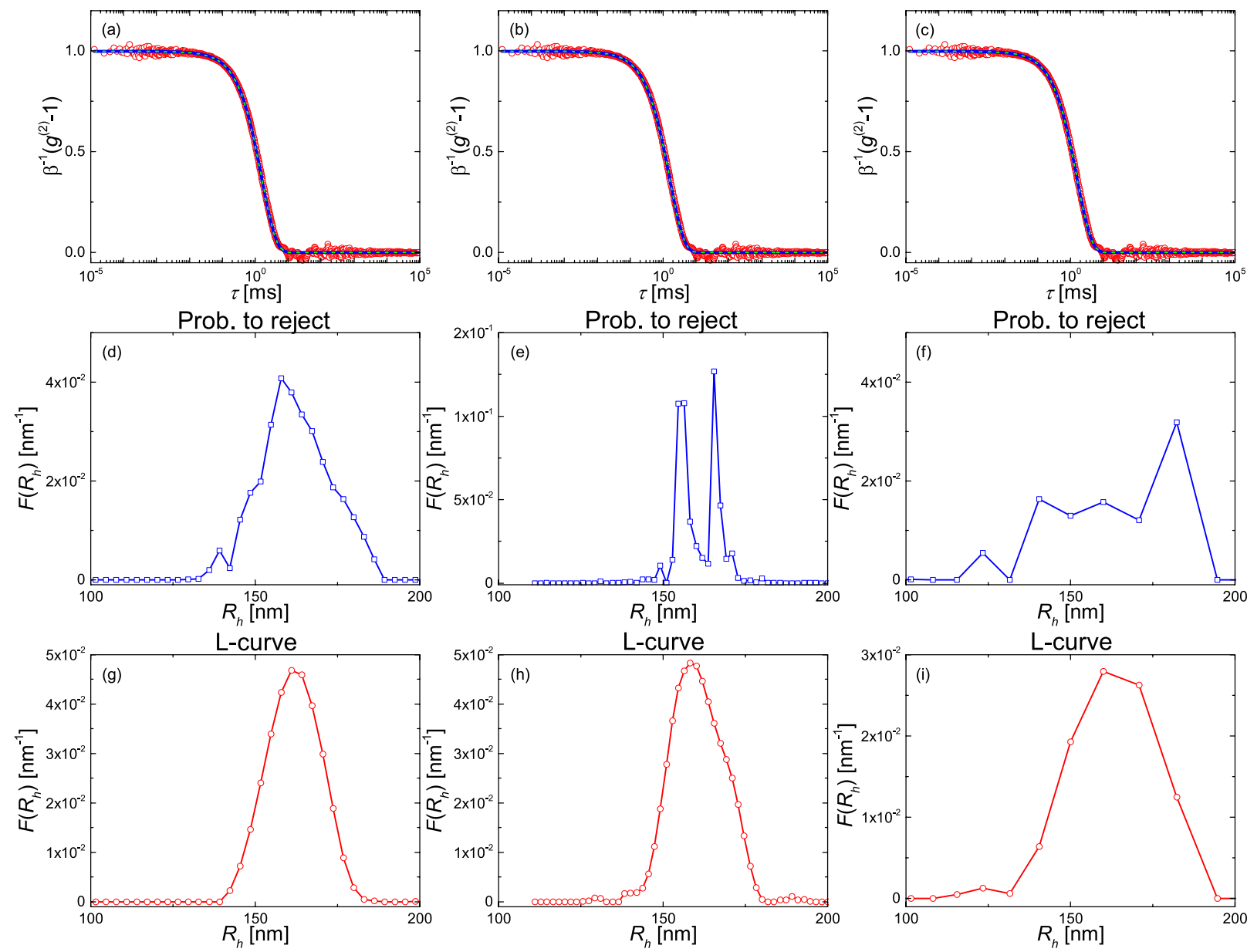

FIG. 8. Data analysis of sample s5 at $\theta=70^{\circ}$ with different integration ranges: (a), (d), (g) [1, 500] nm; (b), (e), (h) [110, 400] nm; (c), (f), (i) [0.15, 5000] nm. (a)-(c) Experimental correlation functions (red circles) and data fits according to an $\alpha$ determined with L-curve (blue line) and probability to reject (green dashed line) criteria. (d)-(f) Size distributions computed with the best $\alpha$ obtained with the probability to reject criterion. (g)-(i) Size distributions computed with the best $\alpha$ obtained with the L-curve criterion. These data sets were acquired over a measurement time of $150 \mathrm{~s}$. For the analysis, we used $N_{g}=160$. In (f) and (i), we used $N_{g}=160$ logarithmically spaced points. Note that panels (d)-(f) and (i) have been plotted with different y-axes. 


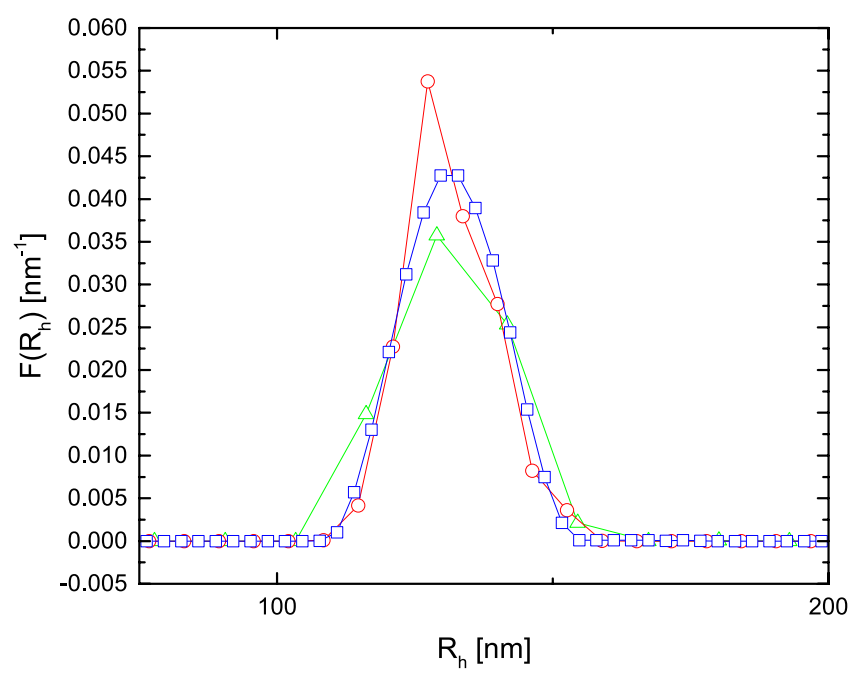

FIG. 9. Size distributions of sample s1 at $\theta=75^{\circ}$ computed using an $N_{g}$ equal to: (green triangles) 40 points, (red circles) 80 points, (blue squares) 160 points.

$N_{g}=40,80$, and 160 to determine the minimum value of $N_{g}$ that gives good resolution in the size distribution. As exemplified in Fig. 9 for sample s1, the mean and width of the distribution do not significantly with the value of $N_{g}$. However, higher values of $N_{g}$ give higher resolution. As a result, we have chosen $N_{g}=160$ for all our analysis.

\section{CONCLUSION}

We have reviewed in detail the CONTIN method for determining the size distribution of microgel suspensions from dynamic light scattering data. We have shown that the Lcurve criterion provides a valid route for determining the regularizer that can be used to invert the field correlation function to obtain the decay rate distribution and hence the particle size distribution. Further, we have shown that the resulting distributions are more stable with regard to noise and varying experimental parameters than the probability to reject method used by the original CONTIN implementation. Our results agree well with those obtained by analyzing SANS data of all microgel suspensions and by performing a cumulant analysis of the DLS data. Though this last method is often used to determine the relative width of the size distribution, careful analysis of the approximations made in this method restrict its applicability to suspensions with sharp size distributions. This emphasizes the need of using regularization methods; however, the side constraint must be based on the physical characteristics of the system under consideration - whether the distribution is monomodal or multimodal, for example. The CONTIN algorithm with the L-curve criterion provides a powerful means to effectively and reliably obtain the size distribution of colloidal suspensions.

\section{ACKNOWLEDGMENTS}

This research was supported by the ACS PRF (No. PRF 50603-DNI7), the Swiss National Science Foundation (No. 200020_153050), and by TAPS International Co., Ltd.

\section{APPENDIX: THEORY FOR NON-RAYLEIGH SCATTERERS}

For a system in the Rayleigh-Gans-Debye or Mie regimes, Eq. (3) is written as ${ }^{29}$

$$
\begin{aligned}
g^{(1)}(q, \tau) & =\frac{\int_{0}^{\infty} d \Gamma G(\Gamma) B^{2}\left(q, R_{h}\right) \exp (-\Gamma \tau)}{\int_{0}^{\infty} d \Gamma G(\Gamma) B^{2}\left(q, R_{h}\right)} \\
& =\frac{\int_{0}^{\infty} d R_{h} F\left(R_{h}\right) B^{2}\left(q, R_{h}\right) \exp (-\Gamma \tau)}{\int_{0}^{\infty} d R_{h} F\left(R_{h}\right) B^{2}\left(q, R_{h}\right)},
\end{aligned}
$$

where $B\left(q, R_{h}\right)$ is the angle-dependent particle scattering amplitude, which can be modeled independently of the CONTIN analysis. For a Rayleigh scatterer, $B\left(q, R_{h}\right)$ is constant and Eq. (A1) simplifies to Eq. (3). Rewriting Eq. (A1) gives

$$
g^{(1)}(q, \tau)=\frac{\int_{0}^{\infty} \frac{d R_{h}}{R_{h}^{2}} G\left(\frac{k_{B} T q^{2}}{6 \pi \eta R_{h}}\right) B^{2}\left(q, R_{h}\right) \exp \left(-\frac{k_{B} T q^{2}}{6 \pi \eta R_{h}} \tau\right)}{\int_{0}^{\infty} \frac{d R_{h}}{R_{h}^{2}} G\left(\frac{k_{B} T q^{2}}{6 \pi \eta R_{h}}\right) B^{2}\left(q, R_{h}\right)} .
$$

The expansion in Eq. (4) is unchanged; however, the definitions of the cumulants themselves change, taking on an angular dependence that complicates the analysis.

The expansion in Eq. (5) is unchanged but Eq. (6) again must take the angular dependence into account, changing the definitions of the cumulants

$$
\begin{aligned}
g^{(1)}(q, \tau) \approx & \exp (-\bar{\Gamma} \tau) \\
& \times \frac{\int_{0}^{\infty} d \Gamma G(\Gamma) B^{2}\left(q, R_{h}\right)\left[1-(\Gamma-\bar{\Gamma}) \tau+\frac{(\Gamma-\bar{\Gamma})^{2}}{2 !} \tau^{2}\right]}{\int_{0}^{\infty} d \Gamma G(\Gamma) B^{2}\left(q, R_{h}\right)} \\
= & \exp \left(-k_{1} \tau\right)\left[1+\frac{k_{2}}{2 !} \tau^{2}\right],
\end{aligned}
$$

where

$$
k_{2}=\frac{\int_{0}^{\infty} d \Gamma G(\Gamma) B^{2}\left(q, R_{h}\right)(\Gamma-\bar{\Gamma})^{2}}{\int_{0}^{\infty} d \Gamma G(\Gamma) B^{2}\left(q, R_{h}\right)}
$$

is the second cumulant, or variance of $G(\Gamma)$. It is worth pointing out that since the Rayleigh scattering criterion depends on the product of $q$ and $R_{h}$, even a system of large particles behaves as a Rayleigh scatterer at low enough values of $q$, which is itself dependent on the wavelength of scattered light and the instrument geometry. Thus, even for a system that is not Rayleigh scattering over the entire accessible $q$ range, the suspension polydispersity can often be obtained from the low $-q$ values of the first and second cumulants: $p^{2}=\lim _{q \rightarrow 0} k_{2} / k_{1}^{2}$.

The problem is still ill-posed. For the perturbation shown in Eq. (8), the corresponding perturbation in $g^{(1)}(q, \tau)$ will be

$$
\begin{aligned}
\Delta g(q, \tau) & \\
= & \frac{\int_{0}^{\infty} d \Gamma[G(\Gamma)+\epsilon \sin (2 n \pi \Gamma)] B^{2}\left(q, R_{h}\right) \exp (-\Gamma \tau)}{\int_{0}^{\infty} d \Gamma[G(\Gamma)+\epsilon \sin (2 n \pi \Gamma)] B^{2}\left(q, R_{h}\right)} \\
& -\frac{\int_{0}^{\infty} d \Gamma G(\Gamma) B^{2}\left(q, R_{h}\right) \exp (-\Gamma \tau)}{\int_{0}^{\infty} d \Gamma G(\Gamma) B^{2}\left(q, R_{h}\right)},
\end{aligned}
$$

with $n=1,2, \ldots$. Applying the Riemann-Lebesgue lemma still implies that $\Delta g \rightarrow 0$ as $n \rightarrow \infty$. Finally, the discretization 
of Eq. (A1) gives

$g^{(1)}\left(q, \tau_{m}\right)=\frac{\sum_{n=1}^{N} B^{2}\left(q, R_{h, n}\right) \tilde{G}\left(\Gamma_{n}\right) \exp \left(-\Gamma_{n} \tau_{m}\right)}{\sum_{n=1}^{N} B^{2}\left(q, R_{h, n}\right) \tilde{G}\left(\Gamma_{n}\right)}$.

We can still define the problem in terms of Eq. (10), with $x_{n}$ $=\left[\tilde{G}\left(\Gamma_{n}\right)\right] /\left[\sum_{n=1}^{N} B^{2}\left(q, R_{h, n}\right) \tilde{G}\left(\Gamma_{n}\right)\right]$ and $A_{m, n}=B^{2}\left(q, R_{h, n}\right)$ $\exp \left(-\Gamma_{n} \tau_{m}\right)$. For $q R_{h} \lesssim 1 / 2$, since $B\left(q, R_{h}\right)$ is constant in $q$, this reduces to the simpler Rayleigh scatterer form. The fully developed form complicates the analysis not only due to the inclusion of the scattering amplitudes but also because the distribution $G(\Gamma)$ appears twice in Eq. (A4).

${ }^{1}$ A. N. St. John, V. Breedveld, and L. A. Lyon, J. Phys. Chem. B 111, 7796-7801 (2007).

${ }^{2}$ H. Senff and W. Richtering, J. Chem. Phys. 111, 1705-1711 (1999).

${ }^{3}$ S. V. Vinogradov, Curr. Pharm. Des. 12(36), 4703 (2006).

${ }^{4}$ S. Auer and D. Frenkel, Annu. Rev. Phys. Chem. 55, 333 (2004).

${ }^{5}$ S. Auer and D. Frenkel, Nature 413, 712 (2001).

${ }^{6}$ U. Gasser, J. Phys.: Condens. Matter 21, 203101 (2009).

${ }^{7}$ Z. Meng, J. K. Cho, S. Debord, V. Breedveld, and L. A. Lyon, J. Phys. Chem. B 111, 6992 (2007).

${ }^{8}$ D. Mcglinchey, Characterisation of Bulk Solids (Oxford, 2005).

${ }^{9}$ J. P. M. Syvitski, Principles, Methods, and Application of Particle Size Analysis (Cambridge University Press, Cambridge, 2007).

${ }^{10}$ A. S. Dukhin and P. J. Goetz, Ultrasound for Characterizing Colloids Particle Sizing Zeta Potential, Rheology (Elsevier Science, The Netherlands, 2002).

${ }^{11} \mathrm{C}$. Solomon and T. Breckon, Fundamentals of Digital Image Processing: A Practical Approach with Examples in Matlab (Wiley-Blackwell, Oxford, 2010).

${ }^{12}$ R. Pecora, Dynamic Light Scattering: Application of Photon Correlation Spectroscopy (Plenum Press, New York, 1985).

${ }^{13}$ W. Brown, Dynamic Light Scattering: The Method and Some Applications (Oxford Science Publications, Oxford, 1993).

${ }^{14}$ D. E. Koppel, J. Chem. Phys. 57(11), 4814-4820 (1972).

${ }^{15}$ N. Ostrowsky, D. Sornette, P. Parker, and E. R. Pike, Opt. Acta: Int. J. Opt. 28, 1059 (1981).

${ }^{16}$ I. D. Morrison and E. F. Grabowski, Langmuir 1, 496 (1985).

${ }^{17}$ M. Bertero, P. Brianzi, and E. R. Pike, Proc. R. Soc. London 398, 23 (1985).

${ }^{18}$ A. N. Tikhonov and V. Arsenin, in Solutions of Ill Posed Problems (V. H. Winston, Michigan, 1977), Vol. 383.

${ }^{19}$ L. M. Gugliotta, G. S. Stegmayer, L. A. Clementi, and V. D. G. Gonzalez, Part. Part. Syst. Charact. 26, 41 (2009).
${ }^{20}$ S. W. Provencher, Comput. Phys. Commun. 27, 213-227 (1982).

${ }^{21}$ I.-G. Marino, rilt, Matlab central (2013).

${ }^{22}$ M. Urbanczyk, D. Bernin, W. Kozminski, and K. Kazimierczuk, Anal. Chem. 85, 1828 (2013).

${ }^{23}$ G. Wahba, Spline Models for Observational Data (SIAM, 1990).

${ }^{24}$ V. A. Morozov, Methods for Solving Incorrectly Posed Problems (Springer, 1984).

${ }^{25}$ W. Liu, X. Sun, and J. Shen, Opt. Laser Technol. 44, 1-5 (2012).

${ }^{26}$ P. C. Hansen, Numer. Algorithms 46, 189-194 (2007).

${ }^{27}$ R. Borsali and R. Pecora, Soft Matter Characterization (Springer Science +Business Media, 2008).

${ }^{28}$ P. N. Pusey, Curr. Opin. Colloid Interface Sci. 4, 177-185 (1999).

${ }^{29}$ J. K. G. Dhont, An Introduction to Dynamics of Colloids (Elsevier, 1996), Chap. 3.

${ }^{30}$ I. S. Gradshteyn and I. M. Ryzhik, Tables of Integrals, Series, and Products, 6th ed. (Academic Press, San Diego, 2000).

${ }^{31} \mathrm{C}$. W. Groetsch, The Theory of Tikhonov Regularization for Fredholm Equations of the First Kind (Academic Press, San Diego, 1984).

${ }^{32} \mathrm{H}$. W. Engl, M. Hanke, and A. Neubauer, Regularization of Inverse Problems (Kluwer Academic Publishers, Dordrecht, 2000).

${ }^{33}$ P. C. Hansen, Numer. Algorithms 6, 1-35 (1994).

${ }^{34}$ X. Zhu, J. Shen, W. Liu, X. Sun, and Y. Wang, Appl. Opt. 49, 6591 (2010).

${ }^{35}$ J. C. Lagarias, J. A. Reeds, M. H. Wright, and P. E. Wright, SIAM J. Optim. 9, 112 (1998).

${ }^{36}$ B. J. Frisken, Appl. Opt. 40(24), 4087-4091 (2001).

${ }^{37}$ A. Ramzi, C. J. F. Rijcken, T. F. J. Veldhuis, D. Schwahn, W. E. Hennink, and F. van Nostrum, J. Phys. Chem. B 112, 784 (2008).

${ }^{38}$ F. Horkay, P. J. Basser, A. M. Hecht, and E. Geissler, Polymer 46, 4242 (2005).

${ }^{39}$ Y. Hirokawa, T. Okamoto, K. Kimishima, H. Jinnai, S. Koizumi, K. Aizawa, and T. Hashimoto, Macromolecules 41, 8210 (2008).

${ }^{40}$ K. Kratz, T. Hellweg, and W. Eimer, Polymer 42, 6631 (2001).

${ }^{41}$ J. J. Lietor-Santos, U. Gasser, R. Vavrin, Z. B. Hu, and A. Fernandez-Nieves, J. Chem. Phys. 133, 034901 (2010).

${ }^{42}$ M. Stieger, W. Richtering, J. S. Pedersen, and P. Lindner, J. Chem. Phys. 120, 6197-6206 (2004).

${ }^{43}$ P. S. Pedersen, D. Posselt, and K. Mortensen, J. Appl. Crystallogr. 23, 321-333 (1990).

${ }^{44}$ I. Berndt, J. S. Pedersen, and W. Richtering, Angew. Chem. 118, 1769 (2006).

${ }^{45}$ S. W. Provencher, "Inverse problems in polymer characterization: Direct analysis of polydispersity with photon correlation spectroscopy," Makromol. Chem. 180, 201-209 (1978).

${ }^{46} \mathrm{H}$. Ruf, B. J. Gould, and W. Haase, "The effect of nonrandom errors on the results from regularized inversions of dynamic light scattering data," Langmuir 16, 471-480 (2000). 